Gooden, Gerald

\section{Determining the quantitative accuracy of CDNA microarrays}

\author{
Gerald Gooden, David M orse, Arthur Glatfelter, Yidong Chen, \\ Jeffrey M. Trent, Paul S. M eltzer \& M ichael Bittner
}

Cancer Genetics Branch, National Human Genome Research Institute, National Institutes of Health, Bethesda, M aryland, USA

Gene expression profiles for large sets of genes can be generated simultaneously with cDNA microarrays. The quantitative accuracy of this assay requires that the immobilized probes be present in abundant, non-limiting amounts relative to the amount of labelled target. When this is true, the rates of hybridization for the cDNA targets assayed will follow pseudo first-order kinetics and be limited only by the relative concentrations of the probes themselves, providing linear increases of signal relative to input target. We have examined how the levels of PCR product in the printing solution and the amounts of labelled cDNA target in the hybridization change net detectability and ratio stability in our assays, and will present statistical evaluations of their effect on reliability.

\section{Gregg, Jeff}

\section{The generation of a brain tumour specif- ic CDNA microarray for molecular profiling of oligodendroglial and astrocytic tumours}

\author{
Jeff Gregg \\ UC Davis School of M edicine, Pathology 4645 Second St, Sacramento, \\ California 95817, USA
}

Gliomas, which represent the second leading cause of cancer death in individuals under age 35 and the fourth leading cause in those over age 54, are a significant medical problem. To date, the diagnostic classification of gliomas has relied on histologic criteria. These histology-based grading systems (WHO and DaumasDuport) are sufficient as general criteria for grouping patients in terms of survival, but are deficient because they often fail to provide information on the individual patient's clinical course and sensitivity to chemotherapy and radiation protocols. In addition, due to the subjective nature of the classification system, difficulties arise in grading tumours and differentiating certain tumour types (e.g. oligodendrogliomas/oligoastrocytomas vs. astrocytomas), which have very different behaviours and responses to chemotherapy. As histologic classification cannot adequately predict tumour behaviour, there arises the need for a better system for classifying oligodendroglial and astrocytic tumors. As human cancer is a consequence of the dysregulation or alteration of the subset of genes expressed, our hypothesis is that the behaviour of a given tumour is encoded within a set of genes expressed and that these genes can be used to better classify tumours, specifically oligodendroglial, astrocytic and mixed oligo-astrocytic tumours, and predict prospective responses to clinical intervention.

We propose to exploit microarray technology for the parallel analysis of gene expression in order to classify brain tumours on a molecular basis. We have created an 8,000-clone oligodendroglial and astrocytic tumour specific brain array to reach this goal. This array has been generated by a comprehensive informatic database search of the Unigene Database (Unigene build \#69). A set of approximately 7,400 non-redundant genes and ESTs were identified in four astrocytic tumour libraries and two oligodendroglioma libraries representing unique clusters in the Unigene Database. An additional 100 genes, not included in the initial search but known to be important in brain tumour biology were included. Five hundred genes, not necessarily expressed in astrocytic or oligodendroglial tumours, but known to be important in tumour biology and expressed in brain, were included. These genes include many tumour suppresser genes that may be aberrantly down-regulated in brain tumours. A unique set of 100 brain tumour "housekeeping" genes, found in both the Soares adult brain library and the normalised Soares infant brain, will also be included. All clones selected for the array have been chosen to include, when possible, the 3' UTR.

These clones, obtained in collaboration with Research Genetics Inc., will be PCR amplified and arrayed in duplicate onto glass slides. Fluorescently-labeled RNA from pure tumour tissue of different histological type and grade will be hybridised to the array. Each tumour will have available extensive clinical information and a selected number of tumours will undergo in vitro chemosensitivity assays. The gene expression profiles will be correlated with the clinical data, tumour chemosensitivity and pathology. These data will be available in a web-based repository. The goal is to demonstrate that molecular classification of brain tumours can provide improved predictors of the clinical behaviour and response to therapy; this will allow appropriate disease management decisions for treatment to be made.

Guilbaud, Cécile

\section{Transcript profiling in b3-adrenergic stimulation of white adipose tissue in mice}

\author{
Cecile Guilbaud $^{1}$, Anna Carlsson ${ }^{1} \&$ Peter Lind $^{2}$
}

${ }^{1} \mathrm{M}$ olecular Biology and Genomics and ${ }^{2}$ Pharmacology Departments, Pharmacia \& Upjohn, Strandbergsgatan 49, P12-4, SE-11287 Stockholm, Sweden

Obesity is a prevalent condition frequently associated with diabetes, hypertension and cardiovascular disease. Because available treatments are minimally effective, substantial efforts have been directed toward the discovery of new and effective anti-obesity drugs. The Beta3-adrenergic receptor (AR) represents one of a number of potential anti-obesity drug targets for which selective agonists have been developed. In rodents, 3-AR mRNA is abundant in white adipose tissue (WAT) and brown adipose tissue (BAT). It has recently been demonstrated that mice lacking endogenous b3-adrenoreceptors have a slight increase in body fat, but otherwise appear normal. These mice are completely resistant to the specific b3-agonist CL-316.243, which has been shown to increase lipolysis, energy expenditure and insulin levels. When the b3-adrenoreceptor was ectopically expressed in WAT and BAT or BAT only, it was demonstrated that the anorectic and insulin secretagogue effects were mediated by WAT. How these effects are mediated by 3-AR agonists remains poorly understood. To investigate the mechanisms by which b3-agonist-stimulated WAT exerts these effects, we have compared the transcript profiles of WAT derived from CL-316.243treated C57BL/6J mice with that of control mice injected with a saline solution. Approximately 200 genes were found to be decreased in the treated animals, and almost 200 genes were found to be increased, among them several key genes known to be involved in obesity. In conclusion, our results confirm that 3 -adrenoreceptors in mouse WAT have a major role in controlling the expression of genes involved in body weight regulation. 